\title{
The 2013 joint ECDC/EFSA report on trends and sources of zoonoses, zoonotic agents and food-borne outbreaks published
}

Eurosurveillance editorial team (eurosurveillance@ecdc.europa.eu) ${ }^{1}$

1. European Centre for Disease Prevention and Control (ECDC), Stockholm, Sweden

Citation style for this article:

Eurosurveillance editorial team. The 2013 joint ECDC/EFSA report on trends and sources of zoonoses, zoonotic agents and food-borne outbreaks published. Euro Surveill. 2015;20(4):pii=21021. Available online: http://www.eurosurveillance.org/ViewArticle.aspx?Articleld=2102

Article published on 29 January 2015

On 28 January 2015, the European Food Safety Authority (EFSA) and European Centre for Disease Prevention and Control (ECDC) joint summary report on trends and sources of zoonoses, zoonotic agents and food-borne outbreaks, was published. The report presents the results of zoonoses monitoring activities carried out in 2013 in 32 European countries, 28 European Union (EU) Member States and four non-Member States [1].

The report shows that campylobacteriosis remains the most commonly reported zoonosis in the EU. After several years of an increasing trend, the campylobacteriosis notification rate has stabilised around the 2012 level. Campylobacter, the causative agent of campylobacteriosis, is mostly found in chicken meat.

The number of reported listeriosis cases, 1,763 , represents an 8.6 percent increase between 2012 and 2013 and reflects an increasing EU trend in 2009-2013. Although the number of confirmed cases is relatively low, it is still a cause for concern as the reported Listeria infections are mostly severe, invasive forms of the disease with higher death rates than for other foodborne diseases.

The number of confirmed verocytotoxigenic Escherichia coli (VTEC) infections in humans also increased. In 2013 reported cases of VTEC infection rose by 5.9 percent compared to 2012. This may reflect the effect of increased awareness in Member States following the 2011 outbreak, which translated into better testing and reporting.

The decreasing EU trend in confirmed human salmonellosis cases observed in recent years continued. The reported number of salmonellosis fell for the eighth year in a row with a 7.9 percent decrease between 2012 and 2013. Most Member States met their Salmonella reduction targets for poultry. The report also shows a continued 2009-2013 decreasing EU trend in confirmed yersiniosis cases. Positive findings for Yersinia were mainly reported in pig meat and pig meat products.
In total, 5,196 food-borne and water-borne outbreaks were reported in the EU in 2013. Salmonella was the most common causative agent in foodborne outbreaks with known origin, followed by viruses, bacterial toxins and Campylobacter. In $28.9 \%$ of all outbreaks the causative agent was unknown. Eggs and egg products, followed by mixed food, and fish and fish products were the most important food vehicles in food-borne outbreaks.

The report further summarises trends and sources along the food chain caused by zoonoses such as Brucella, Trichinella, Echinococcus, Toxoplasma, rabies, Coxiella burnetii ( $Q$ fever), West Nile virus and tularaemia, as well as on cases of tuberculosis caused by Mycobacterium bovis.

Read more about food and waterborne diseases and zoonoses on the ECDC website.

References

1. EFSA and ECDC (European Food Safety Authority and European Centre for Disease Prevention and Control), 2015. The European Union Summary Report on Trends and Sources of Zoonoses, Zoonotic Agents and Food-borne Outbreaks in 2013. EFSA Journal 2015;13(1):3991, 162 pp. doi:10.2903/j.efsa.2015.3991 Available from: http://www.efsa.europa.eu/en/efsajournal/ doc/3991.pdf 